\title{
Espacio, ciberespacio e hiperespacio: nuevas configuraciones para leer la comunicación mediada por computadora
}

\section{Edgar Gómez ${ }^{1}$}

Lo virtual nos ha abierto una nueva perspectiva mental.

Margarita Rivière

\section{Los estudios de Internet}

De Internet se ha hablado mucho y aclarado poco. En principio, una distinción que me parece necesaria es que Internet puede ser visto como algo concreto y unitario, no como un monolito del que se puedan extraer características inamovibles, estructuras predeterminadas y comportamientos predecibles. Incluso si se habla sólo de los cables, computadoras y protocolos que lo sostienen, éstos están desarrollándose de una forma que sería muy difícil definirlos como algo estático. Por mucho tiempo se ha escrito sobre Internet como sinónimo de correo electrónico o de páginas web. Sin embargo, no sólo existen las páginas web (de las que incluso se podría hacer una taxonomía) y el correo electrónico (quizá la parte de Internet que más se utiliza). Internet también son los chats, los juegos en línea, los grupos de noticias, los servidores ftp y muchos más programas, bases de datos y comunicaciones. Aunque existen muchos textos de corte ensayístico y descriptivo sobre Internet, los estudios científicos que se han llevado a cabo sobre éste son en su mayoría específicos acerca de una de estas "partes" que lo componen en relación a su uso. Dichos trabajos están hechos con diferentes marcos epistemológicos (críticos, clínicos, culturalistas), con diferentes enfoques teóricos (desde la

1. Universidad de Colima. Correo electrónico: egomez@cgic.ucol.mx. 
psicología, la telemática, la antropología, la comunicación, etcétera), en países muy diversos, con diferentes lenguajes y hasta en formatos distintos. Esta distinción es importante para diferenciar los estudios que yo en particular he realizado y que tienen que ver con la llamada comunicación mediada por computadora (CMC), ${ }^{2}$ posiblemente la parte más interactiva de Internet. Una definición básica ${ }^{3}$ de ello es: la comunicación que se da entre dos o más personas, utilizando la computadora (conectada a Internet) como medio; estaría hablando entonces de los chats (aunque éstos sean vía web), los llamados mensajeros, los grupos de noticias y el correo electrónico. En estos estudios he repasado cuestiones como la creación de la identidad, la forma en la que socializan las personas, las relaciones afectivas que se crean en la comunicación mediada y, actualmente, el uso sexual de dichos sistemas.

Después de estos intentos por comprender la forma en la que las personas utilizan e integran la CMC en su vida cotidiana, una de las reflexiones que me parece importante y pertinente como punto de inicio, versa sobre Internet como un espacio físico. La discusión dista mucho de estar concluida y de hecho va iniciando. En realidad me parece necesaria porque ésta pudiera ser la clave en una nueva fase en los estudios sobre Internet y la comunicación mediada por computadora.

\section{El espacio, el tiempo y la realidad en un mundo ternológico}

No es mi tarea la de comenzar (o mejor dicho continuar) una discusión sobre la realidad, si ésta se percibe a través de los sentidos, del conocimiento, si es una construcción simbólica, etc. Ya bastante se ha dicho en el pasado, y desde Platón y el mito de la caverna hasta Hegel se ha pensado y escrito bastante sobre ella. Lo que intentaré será una pequeña reflexión sobre el espacio y el tiempo, ya que nuestra concepción de ambos ha sido una herramienta básica para entender, o por lo menos para situar, la realidad, tanto en el plano histórico como en el cartesiano y más allá en la concepción del mundo. Sin embargo, los conceptos de tiempo y espacio con los que habíamos trabajado tradicionalmente se han visto amenazados ante la emergencia de nuevas configuraciones tecnosociales que nos hacen necesaria una reflexión sobre ellos. Ejemplos hay muchos y el texto de Román Gubern $(1991)^{4}$ resulta muy

2. Hago esta distinción para aclarar que, cuando hable de Internet en el resto del texto, me estaré refiriendo exclusivamente a esta parte suya.

3. Para una discusión más extensa y profunda sobre el concepto, cfr. Ess, 1996.

4. Gubern (1991), "La antropotrónica: nuevos modelos tecnoculturales en la sociedad mass- 
ilustrativo al respecto. Siguiendo su reflexión, nos encontramos actualmente con preguntas como: ¿Cómo podemos pensar en la concepción clásica de distancia, en tiempos en que gracias a las telecomunicaciones, con una llamada o la conexión a Internet, se pueden comprar cosas que físicamente están del otro lado del mundo y esto es más sencillo que ir a la tienda de la esquina? $\mathrm{O}$, ¿cómo podemos seguir pensando en el tiempo, en relación a la distancia, tal y como solíamos entenderlo, cuando tardamos más de la casa al aeropuerto que en el vuelo de una ciudad a otra? Necesitamos una nueva configuración para entender estas dos herramientas con mayor flexibilidad. No estoy proponiendo aquí que ambos desaparecerán como conceptos, ni mucho menos, pero sí considero que es necesario otorgarles dimensiones más acordes con la realidad actual en la que se ven inmersos, e incluso dejarlos de tener como marcos estructurales de la realidad.

En esa vía de pensamiento, el concepto de realidad que me parece adecuado no es el de la realidad de los sentidos, sino el de la realidad experiencial y vivencial (aunque evidentemente también la de los sentidos es así), es decir, la que se vive, se siente o se percibe, y que no es necesariamente observable, objetiva y comprobable. Pongo un ejemplo: para sentir miedo no hace falta que exista un peligro "real", basta con que exista la percepción de dicho peligro, y lo que para algunas personas puede ser un elemento de temor, para otras es simplemente un fenómeno más del entorno (las fobias son un buen ejemplo de ello). Esto no significa que el miedo no sea real para la persona que lo siente, pues el corazón se acelera, las sensaciones se afinan y eso tiene consecuencias reales para ella. Un ejemplo más: cuando nos cuentan un chiste, lo que genera la risa no es el hecho observable, sino la decodificación mental de un mensaje que nos remite a ese hecho que, aunque inexistente físicamente en la realidad, genera una reacción real en las personas: la risa.

De esta forma, propongo repensar a Internet en su relación con el espacio tal como lo entendíamos hasta ahora. De esta forma, pensar en el concepto de realidad y su aplicación para estudiar a Internet. De ahí, pasaré al concepto que quiero proponer y terminaré centrándome en la cuestión de la sexualidad mediada por computadora como ejemplo de ello.

\section{Internet: del espacio al ciberespacio}

Al hablar de Internet se habla de realidad virtual, de comunidad virtual y de ciberespacio (cfr. Reinghold, 1995; y Jones, 1995). Aunque en principio pamediática", en Carmen Gómez Mont (ed.), Nuevas tecnologías de comunicación, México,
Trillas. 
rezca que lo que diferencia a Internet de otros conceptos sólo sea lo "virtual" o lo "ciber", aún no queda claro, en el orden teórico, si se habla de un espacio real o de un espacio construido simbólicamente. Bonder (2002: 29) dice que el ciberespacio es un "terreno intangible al que se accede por medios tangibles". En estricto sentido se trata de ambos: por un lado, el espacio que ocupan las computadoras, los cables, las conexiones y los servidores (que es físico, tangible y real) y que está modificando nuestra relación con el espacio físico. Aquí tendríamos un primer objeto de conocimiento, enmarcado en la tradición de los trabajos de Silvertstone, Morley y Hirsch (1992): estudiar la forma en la que se integran al espacio físico estos nuevos dispositivos tecnológicos de comunicación. Cada vez es más común que en las aulas, en lugar de los tradicionales pupitres, se encuentren pequeños muebles con computadoras, y en lugar de salones haya laboratorios de cómputo. En las casas, es relativamente habitual el encontrarnos con el "cuarto de la computadora" o por lo menos con una computadora en algún espacio privilegiado del hogar (Bakardjieva, 2002). Mitra y Schartz (2001) hablan de que, cada vez más, los espacios domésticos se adecuan para que sea más cómoda la entrada al ciberespacio (nuevas conexiones más rápidas, muebles que se integran a la decoración y que son más cómodos, etc.), un punto ampliamente estudiado por Silverstone, Hirsch y Morley (1992). Otra pista de la manera en que Internet está modificando nuestros espacios físicos tradicionales la da el crecimiento de los cibercafés o de los llamados kioscos digitales en las ciudades, lo que demuestra cómo los espacios sociales están adecuándose cada vez más para el acceso al ciberespacio.

Por otro lado, y es precisamente el que me interesa en esta reflexión, está el espacio que posibilitan esta serie de elementos tecnológicos, un espacio simbólico que surge por el uso que hacen las personas de ellos. Como dice Jones (1995: 16) con referencia a la comunicación mediada por computadora (CMC):

La CMC, por supuesto, no es sólo una herramienta; es al mismo tiempo tecnología, medio y motor de relaciones sociales. No sólo estructura relaciones sociales, es el espacio en donde dichas relaciones ocurren y la herramienta que los individuos usan para entrar en ese espacio. Es mucho más que el contexto en donde ocurren esas relaciones (aunque eso sea también). Por ello, es comentado e imaginativamente construido por procesos simbólicos iniciados y mantenidos por individuos y grupos.

De hecho, la misma terminología apunta a pensar en este "(ciber) espacio" como un espacio exterior al que existe en la realidad. Miller y Slater (2001: 4) comentan: 
La clase de preguntas que, de esta manera, han preocupado a la literatura de alto perfil, así como a la mayoría de la discusión pública y el sentido común acerca de la red, han asumido así, una oposición entre lo virtual y lo real.

Se habla entonces de acceder, entrar, conectarse, estar "en línea", y esto nos remite a la sensación de entrar en este espacio cada vez que estamos frente a la computadora y ésta se encuentra conectada a Internet. Diversos autores conciben al mundo de Internet como un espacio físico en sí mismo, tanto en metáforas como en sentido directo: desde el mismo concepto de "ciberespacio", acuñado por Gibson, que describe una alucinación múltiple al acceder mediante una computadora a un espacio. Antulio Sánchez (2001) habla de él como el "sexto continente", Gwinnell (1999) lo compara con un centro comercial gigante, Hine (2000: 30) hace un recuento de ello al decir que Internet se ha concebido como "tienda de conocimiento, medio de comunicación, un foro comercial y un lugar para la experiencia". Y también se ha denominado a Internet como la "frontera electrónica". 5 Nuevamente Miller y Slater (2001: 5) critican esta posición al decir que "esta concentración en la virtualidad o separación como el factor definitorio de Internet podría tener que ver menos con las características de Internet y más con las necesidades de proyectos intelectuales". Y continúan diciendo que Internet representa "nuevas mediaciones, de hecho, pero no una nueva realidad", para concluir:

podríamos ir más lejos aquí: la virtualidad —como la capacidad de las tecnologías comunicativas de constituir en lugar de mediar realidades y de constituir esferas de interacción relativamente limitadas - no es ni nueva ni específica de Internet. En cambio es probablemente intrínseca al proceso de mediación (p. 6).

Esto tendría que pensarse más a fondo. Ahora bien, más allá de metáforas o conceptos para denominar Internet, existen pocas reflexiones teóricas sobre el asunto. Una de ellas, a mi parecer muy interesante, es la propuesta por Castells (2001: 452), quien establece el concepto del "espacio de los flujos" y de él dice: "Las localidades se desprenden de su significado cultural, histórico y geográfico, y se reintegran en redes funcionales o en collages de imágenes, provocando un espacio de flujos que sustituye al espacio en lugares". Con ello, da a las redes comunicativas (en general y a Internet por ser la mayor red tecnológica-comunicativa-humana) una nueva configuración que establece el espacio en la interacción más que en los medios para llevarla a cabo. Luhmann (2000: 24), al hablar de las tecnologías que posibilitaron el espacio simbólico de los mass media (y con ellos el sistema autopoiético que él señala), dice que

5. Que surge de la página www.eff.org, una organización que se dedica a luchar por los derechos de Internet libre. 
"estas tecnologías no sólo ahorran que haya comunicación entre presentes, sino que expresamente, para la comunicación específica de los mass media, la excluyen", y continúa diciendo: "Evidentemente, la comunicación oral permanece como reacción a la comunicación impresa o emitida; sin embargo, el éxito de la comunicación planificada ya no depende de ella". Esta reflexión funciona para los medios masivos de comunicación (y alguna parte de Internet). Morse (1998), en esta misma línea, apunta que lo virtual comenzó con la TV pero llegó a su punto cúspide con lo ciber. De esta forma, la $\mathrm{CMC}$ abre una puerta al sistema e introduce un sentido más complejo y simbólico a la palabra "medio", ya que aunque sigue siendo una construcción tecnológica, los que "generan" el espacio (los contenidos, su intensidad y su dirección) son las personas que tienen acceso al sistema.

\section{Ciberespacio: realidad real y realidad virtual}

Pensemos, por ejemplo, esta reflexión teórica sobre Internet como espacio, comparándolo con los medios de comunicación "tradicionales". Thompson (1998), siguiendo la reflexión de Nowotny, habla de que los medios hicieron posible la "simultaneidad espaciada" ya que antes de éstos, en la comunicación oral, "al mismo tiempo" suponía "el mismo lugar". Es en este punto que se empieza a hablar de una telecomunicación (que de hecho y en términos prácticos, comienza con los tambores hace miles de años). Esta ruptura radical entre los conceptos de tiempo y espacio como definitorios de lo real, comienza con los medios y se ve cada vez más posibilitada por ellos.

Ahora bien, si tomamos como punto de partida al espacio, podemos decir que, por ejemplo, la televisión o la radio son aparatos que permiten reproducir lo que se está llevando a cabo en un espacio lejano; no son vistos como un espacio en sí, en todo caso, son vistos como la referencia o reproducción de otros espacios (los programas de viajes serían un ejemplo práctico). ${ }^{6} \mathrm{El}$ teléfono, las cartas o los faxes tampoco son vistos como espacios diferentes, sino como herramientas para salvar la distancia entre dos personas (o más, con los nuevos sistemas digitales). Es decir, acortar el espacio y a la vez establecer flujos de comunicación sin importar la distancia. Quizá el único de los medios de comunicación que represente un espacio en sí mismo sea el cine, en donde no sólo vemos, como en la televisión, lugares que están lejanos, sino que tenemos que desplazarnos hacia un lugar en particular y con ciertas características para tener acceso a estos mensajes, aunque esa es precisamen-

6. Para una discusión sobre los medios masivos como un sistema en sí mismo, véase Luhmann (2000). 
te su limitante, que nos transporta físicamente a un lugar para, con la oscuridad y la disposición de la sala, sumergirnos en esos mensajes. ¿Por qué es importante esta idea? Porque Internet no sólo representa, como la radio o la televisión, un transmisor de contenidos o una forma de acortar distancias como el teléfono, sino que puede funcionar como un espacio social en sí mismo, como si fuera un café o un parque en donde se citan las personas para interactuar. Además, a diferencia del cine, en el ciberespacio no sólo se "asiste" para recibir mensajes sino también para enviarlos y que esto genere una sensación de "estar" con alguien (que incluso puede ni siquiera estar en línea en ese momento) y, lo más importante, generar un ser que puede no sólo comunicarse sino existir. Como dice Rodríguez (en línea):

\begin{abstract}
Ahora el espectador se introduce en el cuadro, como Alicia en el espejo, y se vuelve actor de lo que allí suceda: es su ser y su hacer, su aquí y ahora, sin solución de continuidad, lo que se desenvuelve en ese decorado electrónico. Pero no sólo eso: no es sólo espectador devenido actor, en el sentido escénico (eso ya lo proporcionaban el teatro con la catarsis y el cine a través de la proyección o la identificación psicológicas con los protagonistas), sino también autor, al tiempo guionista instantáneo e intérprete de su peripecia.
\end{abstract}

Recapitulando, entonces, Internet se concibe no sólo como un medio de comunicación (que además tiene posibilidades tanto sincrónicas como asincrónicas), sino como un espacio social de interacción. De ahí la idea de "citarse", "encontrarse" o "quedar de verse" en el ciberespacio. Por ello, no resulta tan descabellada la idea de pensar en Internet como un espacio físico real, en donde, en principio, el referente podrían ser los lugares de interacción que conocemos (bares, cafés, plazas, etc.), pero que tiene su propia configuración tecnológica y discursiva, lo que Echeverría (2000: 108) llama "el tercer entorno", siendo el primero la naturaleza y el segundo la ciudad. Sobre este tercer entorno, él comenta que: "La posibilidad de crear comunidades virtuales en el tercer entorno es una de las confirmaciones de la hipótesis de que estamos ante un nuevo espacio social, y no simplemente ante un medio de información y comunicación". Aunque una tecnología como los videojuegos, sobre todo con las versiones más nuevas, permite que un usuario juegue en un espacio virtual, no se puede pensar como un espacio social en sí mismo (a menos que dos personas estén jugando, y aun así me parece que la posibilidad de llevar la interacción a donde se desee, más que las limitadas posibilidades que propone el juego, se convierte en una diferencia sustancial). Ahora bien, por un lado vemos que Internet puede pensarse como un espacio diferente del real, un espacio que pareciera ser más seguro, aislado, separado y diverso (con todas las críticas que estas características pudieran suscitar), e incluso se habla de que en Internet se puede representar una persona diferente de sí misma, 
pertenecer a una comunidad global, etc. Sin embargo, el que pensemos a Internet como un espacio alternativo no quiere decir que sea ni autónomo ni necesariamente independiente, por el contrario, de acuerdo con algunos autores, "El comportamiento en la realidad puede ser influenciado por el discurso encontrado en lo ciber, y es la suma de los comportamientos y los discursos lo que necesita estudiarse de manera conjunta cuando se mira al espacio cibernético" (Mitra y Schartz, 2001, en línea). Éste es un punto de ruptura muy importante con algunos de los trabajos primarios sobre la CMC, que también otros autores señalan (cfr. Miller y Slater, 2001), ya que se transforma la idea de que el ciberespacio es un espacio independiente y por lo tanto se estudia aislado: "Examinar sólo lo virtual ofrece una mirada parcial de un fenómeno que está actualmente llevándose a cabo dentro del espacio cibernético construido en sí mismo por la intersección de lo real con lo virtual" (en línea). En realidad no se ha podido establecer un marco teórico de análisis de dichos fenómenos y se ha caído en la trampa de utilizar conceptos construidos para otros fines y tratar de entender con ellos nuevos fenómenos. Nuevamente Echeverría (2000: 117) señala que:

Carecemos de formación para movernos e intervenir en los escenarios virtuales. Hemos sido educados para expresarnos plausiblemente en los escenarios del primer y segundo entorno, pero ignoramos casi todo sobre la estructura del espacio telemático, ya que estamos acostumbrados a pensarlo como un espacio informacional y no como un espacio de acción.

Ahora, si aceptamos la idea de que el espacio y el ciberespacio son dos mundos que pueden ser distintos, aunque sólo sea para ponerla a prueba, ¿cómo es la separación entre estos dos mundos?, iexiste interdependencia?, ison paralelos?, ise afectan? Graham (2000: 161), al hablar del concepto de comunidad virtual, señala que ésta:

Puede ser considerada como un modo distinto de existencia que no es simplemente un juego, sino un mundo propio, en el que es posible lograr y perder un número importante aunque limitado de cosas. Si ahora añadimos que Internet está en una etapa muy temprana de desarrollo, existen razones para pensar que el futuro del ciberespacio aportará novedades metafísicas y que la realidad virtual interpretada a través de la comunidad virtual es, de algún modo, un mundo nuevo a cuyas puertas estamos llegando.

Es decir, y aquí coincido con el autor, se han tratado de establecer símiles entre situaciones de la vida real y situaciones en el ciberespacio que han dado lugar a discusiones sobre su correcta aplicación, conceptos como: comunidad virtual, comunicación masiva, realidad virtual, que son términos que se han desarrollado en ámbitos diferentes y luego han sido usados para establecer 
analogías que después han sido duramente criticadas. Sin embargo, me parece que la concepción de Graham sobre una realidad diferente de la que vivimos cotidianamente puede ser útil. No es que el ciberespacio no tenga referentes constantes y totales en la vida real, no es que las personas dejen de ser reales cuando entran a este espacio, no es que no haya conexión alguna, la propuesta es que se trata de un mundo paralelo que se alimenta del mundo real, pero que a su vez lo retroalimenta, y que sin embargo puede experimentarse separado en algunas ocasiones. Como dice Maldonado (1999: 40):

Ver presupone saber, la experiencia de percibir un objeto (y aprehender o no su significación) está disolublemente vinculada con la experiencia tenida (o no tenida) antes con ese objeto. Pero las cosas se complican cuando se trata de percibir un objeto desconocido que no ha sido presentado antes, sino que está representado. En este caso es decisiva la familiaridad que tenga el observador con el uso del medio en representación, porque cuando esa familiaridad es escasa o falta en absoluto las cosas pueden complicarse.

Entonces, siguiendo esta reflexión, podríamos hablar de dos mundos (que podríamos llamar real y virtual) que conviven con diferentes percepciones y apropiaciones por parte de las personas. Es decir, nosotros tenemos asumido cognitivamente nuestro referente de lo real, lo tangible, lo que los sentidos nos dicen que existe. Esta realidad puede ser mediada e incluso creada. Luhmann (2000: 77), hablando del entretenimiento en los medios masivos, dice:

En lugar de ello el corte que se produce en la realidad y mediante el cual se constituye un segundo mundo se lleva a cabo mediante una marca acústica u óptica: como libro, como pantalla, como secuencia de un ruido que, en este sentido, se percibe como "tono". Este espacio exterior hace surgir un mundo libre en el cual se inserta la propia realidad ficticia: un mundo - y no únicamente, como en los juegos sociales, una secuencia social de comportamiento coordinado.

Y continúa diciendo: "El entretenimiento hace surgir objetos reales autoproducidos; por así decir, objetos duplicados que posibilitan el cruce de las fronteras entre la realidad real y la realidad ficticia" (p. 77). Él mismo, en este análisis, señala que el teatro renacentista ya lograba esta "recreación entre la alucinación y lo visible, entre el conocimiento y el desconocimiento, entre una presentación conducida por motivos visibles y, al mismo tiempo, por la sospecha generalizada de la autenticidad de dicha motivación" (p. 81). Esto con referencia al entretenimiento; quizá es más clara la realidad en el formato de noticias, por ejemplo. Así, si algo aparece en televisión, en realidad existe (aunque esta mediación tiene cada vez más referentes simbólicos no reales), y aunque no exista en la realidad (en el caso del entretenimiento) existe como símbolo o como ficción (la teoría literaria sobre la ficción es muy interesante 
en este punto). Ahora bien, el mundo del ciberespacio plantea una trampa para la forma en la que percibimos el mundo, ya que no es una copia de éste, ni siquiera una referencia (aunque también lo pueda ser en algunas ocasiones), sino una construcción independiente de un mundo (o muchos mundos) posibles, pero que a la vez no son ficción necesariamente. La vida en el ciberespacio no aspira a ser tan buena (o mala) como la cotidiana; se trata simplemente de una forma de vivencia, con una nueva mediación, un nuevo instrumento y un nuevo insumo. Por ello, me parece que se ha cometido un error (más por la premura de establecer símiles, o por la intención de explicar de alguna forma el fenómeno, que por un análisis erróneo) al nombrar, o tratar de hacerlo, a Internet con conceptos creados para otros fines (y que incluso, como en el caso de "comunidad", siguen estando en discusión). En un trabajo anterior (Gómez, 2000) proponía el concepto de "extensión virtual de la comunidad" en vez de comunidad virtual. Me parece que también estaba equivocado porque no tomaba en cuenta la posibilidad de Internet de ser un espacio con sus propias dinámicas independientes de la cotidianeidad de los usuarios y que, si bien sí puede ser una extensión solamente, puede representar un mundo totalmente independiente, por ejemplo en los MUD. ${ }^{7}$ Tendríamos así dos nuevos objetos de conocimiento: por un lado la relación real de las personas con un medio como es la computadora (lo que algunos autores denominan como identidad vía pantalla), y por otro, las relaciones que se dan en el ciberespacio con independencia de sus condiciones de gestión.

\section{Del mundo al hipermundo, y del ciberespacio al hiperespacio}

Ahora bien, como decía anteriormente, se podría cometer el error de pensar en dos espacios, en dos mundos separados, el de lo real y el de lo virtual, como lo han hecho diferentes autores. Dada esta discusión, que me parece vital para el estudio y entendimiento de Internet, especialmente de la CMC y su verdadero impacto social, propongo el concepto de hiperespacio para buscar entender la relación entre lo virtual y lo real. Este concepto busca englobar la percepción de lo real, de lo ciber y, lo más importante, la suma de ambos. Este concepto se plantea como un vehículo para tratar de entender la complejidad de una tecnología que es (o puede ser) a la vez tecnología, espacio virtual (y extensión del real), símbolo, laboratorio social, etc. Autores como Mitra y Schartz (2001) ya habían avanzado en esta dirección al hablar de "espacios

7. Sistemas basados en texto y programación en los que se crean "mundos virtuales". 
cibernéticos", que "deben ser entendidos como un sistema en su totalidad que puede tener un fuerte componente real y ciber, donde ninguno merece ser privilegiado pero ambos necesitan ser examinados juntos para entender cómo operan los espacios combinados" (Mitra y Schartz, 2001, en línea). La cuestión se complica, porque dentro de este hiperespacio los mundos que lo componen pueden tener concepciones no sólo distintas espacialmente sino también temporalmente, y no sólo como percepciones sino como acciones en sí mismas (cómo lo ven los usuarios y cómo lo estudiamos los investigadores). De ahí, la necesidad de estudiar las transformaciones físicas de los espacios, los tiempos y las percepciones que sobre la realidad tienen los usuarios de Internet y su desenvolvimiento en el ciberespacio. Pero más aún, la convergencia de estos dos puntos y sus diversas relaciones. Nuevamente los autores apuntan: "La buena vida ya no está sólo en lo real, o sólo en lo virtual, sino en la congruencia de los dos, donde uno parece alimentarse dentro del otro, transformando a ambos, y creando el espacio cibernético que se convierte en la síntesis de ambos" (Mitra y Schartz, 2001, en línea).

Dos conceptos pueden ser una buena base para el estudio del hiperespacio. Por un lado está el de "domesticación", que se refiere al proceso de cómo la tecnología se incorpora a la cotidianidad y va transformando los hábitos y los espacios. Y por otro, está el de la "virtualización" del hogar (o del espacio de conexión, que en países latinoamericanos sigue siendo mayor en los centros de cómputo de las escuelas o en cibercafés que en los hogares), esto es, que ya el espacio físico se ve superado por las actividades que se realizan frente a una computadora y que van adquiriendo dinámicas propias con relación al espacio (Green, 2000). Bakardjieva (2002), siguiendo los trabajos de Silverstone, Morley y Hirsch (1992), habla de la micro-regulación de Internet en el espacio doméstico:

Esta regulación tiene que ver con tomar decisiones acerca de temas como el lugar, el acceso, las preferencias, las actividades prohibidas y no recomendadas en, y, a través de Internet y la asignación de recursos, incluidos tiempo, espacio, dinero, atención, etcétera (en prensa).

Esto genera preguntas sobre la forma en que las nuevas tecnologías transforman el entorno cotidiano, y por otro lado, de cómo transformamos la sensación espacial cuando estamos conectados, por ejemplo, al sitio web del Louvre en Francia, o cuando recibimos un correo de algún familiar en Estados Unidos o hacemos una compra en una tienda europea. Esta doble vía, de domesticación de lo tecnológico y de virtualización de lo espacial, genera una relación estrecha entre ambas y una interiorización de dicha dualidad en una forma cognitiva. Por ello, el concepto de hiperespacio puede resultar útil no sólo para hablar de Internet y su impacto en las relaciones sociales y persona- 
les, sino para estudiar dicho impacto desde un acercamiento más claro y preciso, ya que combina, de forma holística, tanto la realidad que vive la persona como la llamada realidad virtual, que también es experimentada. Pero sobre todo, el flujo que, entre ambas, tiene una forma específica de apropiación por parte de los usuarios y usuarias.

El tiempo también es otro factor interesante en este hiperespacio y digno de considerarse en relación a Internet, ya que al ser posible que sea un medio sincrónico o asincrónico, esto transforma nuestras concepciones sobre la realidad. Nuevamente, Castells (2001: 452) apunta que:

El tiempo se borra en el nuevo sistema de comunicación, cuando pasado, presente $y$ futuro pueden reprogramarse para interactuar mutuamente en el mismo mensaje. El espacio de los flujos y el tiempo atemporal son los cimientos materiales de una nueva cultura, que trasciende e incluye la diversidad de los sistemas de representación transmitidos por la historia: la cultura de la virtualidad real, donde el hacer creer acaba creando el hacer.

Esta cita me parece que concretiza y apuntala el sentido del concepto de hiperespacio, ya que le da al ciberespacio una existencia en sí mismo, sin que esto signifique que dicha existencia esté separada de la realidad. Le da una certificación (para estar a tono con el lenguaje empresarial que caracteriza la modernidad), la posibilidad de coexistir con la realidad (o las realidades), dándole un carácter simbólico y sin perder de vista la relación con la otra realidad. Por ello, se establece la necesidad de pensar sobre nuestra concepción del mundo y de la realidad con una mirada más compleja, y con ello poder establecer un nuevo marco organizador de nuestro entendimiento sobre ésta (y de paso para poder estudiar a Internet sin caer en el error de creer que es una "copia" del mundo real y palpable), en donde el concepto de hiperespacio podría funcionar como este marco organizador.

Ahora bien, existen voces menos optimistas en torno a esta mediatización de la realidad: Nora (1995: 69) habla de que el ciberespacio "se ha convertido así en espejo deformante de la vida real, el LSD de los años 90 "; por su parte, Subirats menciona que:

las formas de percepción de la realidad y de interacción comunicativa mediadas por los sistemas de comunicación e información electrónica señalan en una dimensión nueva y diferente. No solamente se trata del empobrecimiento de la experiencia humana o de la "desrealización" del sujeto. Se trata también de su sustitución por las técnicas y estética de producción de la realidad (2001: 14).

En general, me parece que la percepción de muchas personas en torno a la comunicación mediada por computadora se fúnde con un mar de información sobre lo que son y representan las nuevas técnologías, sobre todo si no 
son estudiosas del tema. Nuevamente nos encontramos con el problema de las terminologías y la forma en la que se difunden éstas; por ejemplo, Maldonado (1999: 76), al hablar de "realidad virtual", dice que:

Las realidades virtuales deshacen nuestro vínculo con el mundo de las cosas y de los cuerpos, disminuyen cada vez más nuestras posibilidades de experiencia con el universo físico. No podemos sin embargo olvidar que estas construcciones icónicas se elaboran sobre la base de nuestra experiencia pasada y presente con este mundo y con este universo.

En ese sentido él establece la relación entre la experiencia del mundo y la vivencia de éste nuevo, pero su análisis se centra en la realidad virtual gráfica, de inmersión, no sobre la realidad virtual que representa el ciberespacio. En este sentido, es importante la distinción, porque en la realidad virtual no se establece otro mundo, sino que se suplanta el que vivimos por uno generado en la computadora, de manera que el hiperespacio no se enriquece ni se incrementa, como sucede con el ciberespacio, sino que sólo se simula un nuevo espacio real.

Me parece importante la reflexión sobre Internet y la noción del espacio que genera para futuros marcos teóricos en su estudio y de la CMC. Pienso que precisamente esta delimitación podría ser de utilidad al tratar de entender la forma en que las personas utilizan, se apropian y le dan sentido a estas nuevas herramientas tecnológicas que son a la vez simbólicas y sociales.

Por un lado, tenemos entonces que se puede estudiar la transformación que viven los espacios físicos por el uso de las nuevas tecnologías, especialmente las computadoras e Internet. Por otro, tenemos la relación que existe entre las personas que utilizan Internet y la virtualidad, la manera en la que interactúan físicamente con el espacio virtual. Podemos estudiar también, únicamente, las dinámicas sociales dentro de la virtualidad y la forma en la que se dan éstas. Sin embargo, para que un análisis quede completo y aspire a ser un acercamiento más complejo y holístico tienen que tomarse en cuenta todas estas relaciones. Por ello, introduzco el concepto de hiperespacio como marco organizador de esta reflexión y propongo que es precisamente en este hiperespacio en donde se vive la cognición sobre lo social; por lo tanto, en las investigaciones sobre la CMC no se debe dar una importancia mayor a lo virtual (ciberespacio), o bien a lo real, sino justamente a la relación entre ambos y a la vivencia conjunta por parte de los usuarios y usuarias. Esta propuesta teórica surge de algunos años de trabajo empírico y pretende ser una aportación a la discusión sobre el imfacto social de Internet. 


\section{Bibliografía}

Bakardjieva, M. (2002), "Taming a Wild Medium: The Microregulation of the Internet", ponencia presentada en la IV International Conference Crossroads in Cultural Studies.

Bonder, G. (2002), Las nuevas tecnologías de información y las mujeres: reflexiones necesarias, Chile, CEPAL.

Castells, M. (2001), La galaxia Internet, España, Plaza \& Janés.

Echeverría. J. (2000), Un mundo virtual, España, Plaza \& Janés.

Ess, Ch. (ed.) (1996), Philosophical Perspectives in Computer-Mediated Communication, EE.UU., State University of New York Press.

Gómez, E. (2002), Hacia la construcción de una metodología para el estudio de las comunidades virtuales, en Versión, México, UAM-Xochimilco, núm. 12.

Graham, G. (1999), Internet. Una indagación filosófica, España, Frónesis.

Gubern, R. (1991), "La antropotrónica, nuevos modelos tecnoculturales de la sociedad massmediática", en Carmen Gómez Mont (ed.), Nuevas tecnologias de comunicación, México, Trillas.

Green, L. (2001), Communication, Technology and Society, Londres, Sage.

Gwinell, E. (1999), El amor en Internet. Intimar con desconocidos a través del ciberespacio, España, Paidós.

Hine, Ch. (2000), Virtual Etnography, Londres, Sage.

Jones, S. (ed.) (1995), Cybersociety: Computer-Mediated Communication and Community, EE.UU., Sage Publications.

Luhmann, N. (2000), La realidad de los medios de masas, México, AntrophosUIA.

Maldonado, T. (1999), Lo real y lo virtual, Barcelona, Gedisa.

Miller, D. y D. Slater (2001), The Internet: An Etnographic Aproach, Reino Unido, Berg.

Mitra, A. y R. L. Schartz (2001), "From Cyber Space to Cybernetic Space: Rethinking the Relationship between Real and Virtual Spaces", en Journal of Computer Mediated Communication (en línea), http:www. ascusc.org/jcmc/isseue1/mitra.htaml.

Morse, M. (1998), Virtualities. Televisión, Media Art, and Cyberculture, EE.UU., Indiana University Press.

Nora, D. (1995), La conquista del ciberespacio, España, Andrés Bello.

Rheingold H. (1994), The Virtual Community, Nueva York, Harper Collins.

Rodríguez, R. (2001), Virtualmente suyo, en Telos (en línea), http://www.campusred.net/telos. 
Sánchez, A. (2001), La era de los afectos en Internet, México, Océano.

Silverstone, R., E. Hirsch y D. Morley (1992), "Information and Communication Technologies and the Moral Economy of the Household", en Roger Silverstone y Eric Hirsh (eds.), Media and Information in Domestic Spaces, Nueva York, Routlegde.

Thompson, J. (1998), Los media y la modernidad. Una teoría de los medios de comunicación, México, Paidós. 